\title{
Women's experience of pregnancy and birth during the COVID-19 pandemic: a qualitative study
}

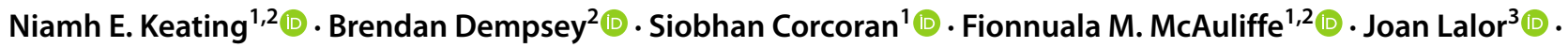 \\ Mary F. Higgins ${ }^{1,2}$ (1)
}

Received: 20 July 2021 / Accepted: 16 November 2021 / Published online: 2 December 2021

(c) The Author(s), under exclusive licence to Royal Academy of Medicine in Ireland 2021

\begin{abstract}
Background The COVID-19 pandemic has changed how maternity care services are provided worldwide. To contain the virus, many providers reduced the number of face-to-face visits for women. In addition, partner attendance was prohibited in many circumstances to protect staff, and other service users, from potential infection.

Aims To explore women's experience of pregnancy and birth in the Republic of Ireland during the COVID-19 pandemic. Methods A qualitative study with 14 women was conducted using a grounded theory approach. Data were collected between April and July 2020, and in-depth interviews were conducted either in pregnancy or in the first 12 weeks after the birth.

Results Six categories emerged: loss of normality, navigating "new" maternity care systems, partners as bystanders, balancing information, uncertainty, and unexpected benefits of pregnancy during the pandemic. While benefits were reported (working from home and additional time spent with partners during the "fourth trimester"), in general, the themes were of increased anxiety and uncertainty.

Conclusion The pandemic caused additional anxiety for pregnant women. This was exacerbated by uncertainty about the effects of COVID-19 on pregnancy and unclear messaging about restrictions. More interactive and personalized communication is required to support women to cope with uncertainty during a pandemic. The birth partner plays an important role as an advocate for women and excluding them from pregnancy care caused additional anxiety for pregnant women. Containment strategies for a pandemic should be developed with this in mind, to view the family as a unit rather than the woman in isolation.
\end{abstract}

Keywords COVID-19 $\cdot$ Experiences $\cdot$ Ireland $\cdot$ Qualitative $\cdot$ Pregnancy $\cdot$ Women

\section{Introduction}

With the declaration of COVID-19 as a Public Health Emergency in January 2020 [1], maternity services had to adapt quickly and change how care was provided. The time-sensitive nature of maternity care meant that the cancellation of services was not an option. Changes made included reducing

Mary F. Higgins

Mary.higgins@ucd.ie

1 Obstetrics and Gynaecology, National Maternity Hospital, 2, Dublin, Ireland

2 School of Medicine, UCD Perinatal Research Centre, University College Dublin, National Maternity Hospital, 65-66 Lower Mount Street, Dublin D02 YH21, Ireland

3 School of Nursing and Midwifery, Trinity College Dublin, Dublin, Ireland the number of face-to-face hospital visits, asking partners and visitors to not attend routine appointments and ultrasound assessments, and placing strict visiting restrictions in postnatal wards and neonatal intensive care unit (NICU) [2].

Early data on the COVID-19 infection in pregnancy was reassuring [3]; however, initial studies were based on small numbers of cases in China, and there was much uncertainty about the potential impact of the virus on pregnancy, particularly in the early stages of the pandemic. Previous coronavirus health crises were associated with significant morbidity including severe respiratory illness, increased intensive care unit (ICU) admission, and mortality in pregnancy [4]; later research showed that COVID-19 infection in pregnancy is also increased with significant maternal and fetal morbidity [5].

This pandemic has been dubbed an "info-demic" [6], and while the H1N1 pandemic of 2009 was the first of the 
internet age, social media plays an even greater role in the rapid spread of information, and at times misinformation, about COVID-19. Perceived mixed messages from public health officials can lead to confusion, uncertainty, and fear [7]. While studies have shown that exposure to media or public health-focused advertising during the H1N1 pandemic had a positive impact on health knowledge and behaviors, the impact of social media platforms such as Twitter and Facebook at that time was not fully evaluated [8].

"Lockdown," by which people were ordered to stay in their homes to reduce social contacts and limit the spread of COVID-19, was a strategy adopted by many countries. Social isolation can have profound effects on mental health, and previous studies have identified women and mothers of children under the age of 18 to be particularly vulnerable [9]. In times of disaster, women in the pre- and postnatal period may experience higher rates of mood disorders than the general public [10]. Those with underlying mental health problems are at risk of post-traumatic stress disorder [11]. After the SARS health crisis, an increase in avoidance behaviors, absenteeism from work, and excessive hand washing was observed [12]. Studies have shown high levels of depressive symptoms and generalized anxiety among pregnant and breastfeeding women during the COVID-19 pandemic in Europe [13, 14].

While pregnancy is a happy time for many, it can be a time of heightened anxiety and uncertainty [15]. Our aim was to understand the lived experience of pregnancy during the pandemic and the effects on the women's home life and social support system as well as learn from the effects that the hospital restrictions and measures had on women and their families.

\section{Methods}

\section{Qualitative approach}

A qualitative study was undertaken using a grounded theory approach to explore women's direct experiences of care during pregnancy and birth and to understand the impact of practices changes due to COVID-19 restrictions on the same. This paper is written in accordance with the Standards for Reporting Qualitative Research (SRQR) [16].

\section{Participants and recruitment}

Recruitment was open to women of any gestation or those who had given birth since the first lockdown was announced (March 27, 2020), attended obstetric- or midwifery-led antenatal care, were residents of Ireland, had not been diagnosed with COVID-19, and were aged over 18. Participants were invited to participate in the study by posters and handouts in clinics and on social media (Facebook and Twitter). Recruitment started in April 2020 and ran until July 2020.

\section{Setting}

This study took place in the metropolitan area of Dublin, Ireland, with all participating women attending one of the three tertiary-level maternity units serving the city. The interviews for this study were conducted between April and July 2020, during the height of, and through to the easing of the first lockdown restrictions. Our research team consisted of four obstetricians, one psychologist, and one academic midwife. None of the clinical staff interviewed women that they were directly involved in providing care to.

\section{Data collection and management}

Data were collected through individual interviews, conducted by either NK or MH. In line with restrictions, interviews were carried out via secure, encrypted video conference (Zoom) or telephone call with digital recording. Participants chose their preferred option. Interviews ranged from 21 to $64 \mathrm{~min}$. Open questions were used initially to elicit women's experiences and views of their care during the restrictions. As the interviews progressed, they became more semi-structured in nature and theoretical sampling and verification of categories progressed. Interviews began with open questions about how their pregnancy/postpartum period has been so far and then moved on to how the pandemic has changed how they envisioned their pregnancy, the impact of the pandemic on the care they received, and how the pandemic has affected their partners/families experience of their pregnancy.

Recordings were transcribed verbatim by two of the researchers (NK and $\mathrm{MH}$ ). During the transcription stage, anonymization was carried, removing any potentially identifying information. Once anonymization was complete, audio recordings were destroyed.

\section{Data analysis}

Data were analyzed using the constant comparative method [17]. Once data are collected, they were analyzed into initial categories. Detailed notes were made after each interview which allowed for interviews to be linked to analysis already performed. Interviews were carried out by two members of the team, keeping the other team members "blinded" thereby maximizing theoretical sensitivity and rigor in the analysis. After each interview was transcribed, it was analyzed which informed the development of questions for the next interview. This was continued until theoretical saturation occurred. One researcher (NK) reviewed all transcripts, analyzing them into categories, with at least one other member 
Table 1 Participant demographics

\begin{tabular}{ll}
\hline Characteristics & Number \\
\hline Age & 4 \\
$24-34$ & 10 \\
$35-45$ & \\
Parity & 9 \\
Primiparous & 5 \\
Multiparous & \\
Ethnicity & 12 \\
White Irish & 2 \\
White British & \\
Pregnancy status at time of interview & 8 \\
Currently pregnant & 6 \\
Had given birth since March 2020 & \\
Planned place of birth & 13 \\
Hospital & 1 \\
Home & \\
Model of antenatal care & \\
Public, consultant lead & 7 \\
Private, consultant lead & 5 \\
Midwifery lead & 2 \\
\hline
\end{tabular}

of the team $(\mathrm{MH} / \mathrm{BD} / \mathrm{SC} / \mathrm{JL})$ providing a second review for each transcript. The research team met after each interview was analyzed in order to ensure rigor within the process. If discordance on categories was present, a discussion took place between the researchers to reach a consensus and reduce the potential for bias. Three women requested copies of their interview transcripts but did not suggest any changes to be made before or during analysis.

\section{Ethical statement}

Ethical approval was granted by the National Maternity Hospital Research Ethics Committee (EC 14.2020). Women were provided with written information, and informed consent was obtained from all participants prior to participation. Consent forms were collected by email in advance with verbal consent again being obtained prior to beginning the interview and prior to any recording taking place.

\section{Results}

Sixteen women contacted the research team expressing their interest and fourteen consented to participate in the study and were interviewed. Of these, two women elected to be interviewed over the phone with the remaining twelve interviews being carried out by the recorded video conference. Demographic information is shown in Table 1, with participant details in Table 2. Six categories were identified as outlined in Table 3 and described in further detail below.

Women described the "new normal" of a pregnancy during a pandemic. They found it difficult to engage with other

Table 2 Participant details

\begin{tabular}{|c|c|c|c|c|}
\hline $\begin{array}{l}\text { Par- } \\
\text { ticipant } \\
\text { number }\end{array}$ & Ethnicity & $\begin{array}{l}\text { Time of } \\
\text { inter- } \\
\text { view }\end{array}$ & Antenatal/postnatal & History \\
\hline 1 & White Irish & July & 3 months postnatal & $\mathrm{P} 0$, emergency Cesarean section in labor for fetal distress \\
\hline 2 & White Irish & July & 3 months postnatal & $\begin{array}{l}\text { P0, postnatal, spontaneous vaginal delivery, Meconium aspiration, neonatal intensive } \\
\text { care admission for } 2 \text { weeks }\end{array}$ \\
\hline 3 & White Irish & July & 39 weeks' gestation & $\begin{array}{l}\text { P1, previous spontaneous vaginal delivery (SVD), interview } 1 \text { week before planned } \\
\text { induction of labor }\end{array}$ \\
\hline 4 & White Irish & July & 13 weeks' gestation & $\begin{array}{l}\text { P1- previous termination of pregnancy for fetal anomaly, in vitro fertilization (IVF) } \\
\text { cycle cancelled due to pandemic but conceived spontaneously }\end{array}$ \\
\hline 5 & White Irish & July & 3 months postnatal & Postnatal, baby underwent surgery for pyloric stenosis \\
\hline 6 & White, UK & July & 35 weeks' gestation & $\mathrm{P} 0$, partner is non-native English speaker \\
\hline 7 & White Irish & July & 28 weeks' gestation & P0, no medical history \\
\hline 8 & White Irish & July & 3 months postnatal & $\begin{array}{l}\text { P1, previous difficult delivery, changed care to plan for homebirth at } 37 \text { weeks due to } \\
\text { pandemic }\end{array}$ \\
\hline 9 & White, UK & July & 28 weeks' gestation & P0, Living in Ireland for one year, private antenatal care \\
\hline 10 & White Irish & July & 8 weeks post-natal & $\begin{array}{l}\text { P0, IVF pregnancy, severe pelvic girdle pain, spontaneous vaginal delivery, private } \\
\text { consultant lead care }\end{array}$ \\
\hline 11 & White Irish & April & 34 weeks' gestation & P0, same sex couple, IVF pregnancy \\
\hline 12 & White Irish & May & 21 weeks' gestation & P0, Clomid pregnancy, interview before anatomy scan \\
\hline 13 & White Irish & June & 2 weeks postnatal & $\begin{array}{l}\text { Previous pre-term delivery, cervical cerclage at } 14 / 40 \text { delivered by spontaneous vaginal } \\
\text { delivery at term }\end{array}$ \\
\hline 14 & White Irish & May & 31 weeks' gestation & $\mathrm{P} 0$, gestational diabetes mellitus \\
\hline
\end{tabular}


Table 3 Categories and subcategories of results

\begin{tabular}{ll}
\hline Categories of results & Sub categories \\
\hline Loss of normality & $\begin{array}{l}\text { Disruption to the normal celebration of } \\
\text { Lack of peer support } \\
\text { Families unable to share in pregnancy }\end{array}$ \\
& \\
Navigating the "new" & $\begin{array}{l}\text { Depersonalization of care } \\
\text { maternity care }\end{array}$ \\
system & $\begin{array}{l}\text { Fisruption to normal antenatal visits } \\
\text { Benefit of early transfer home } \\
\text { Changes to antenatal education } \\
\text { Difficulty of neonatal care restrictions }\end{array}$
\end{tabular}

Partners as bystanders Loss of reassurance of partner's presence Loss of advocate Impact of restrictions on couple as a family unit
Balancing information Information had the potential to empower but also overwhelm

Unclear language from hospital and national public health department

Lack of up-to-date pregnancy information

Reliance on social media in place of traditional information sources

Uncertainty

Conflicting information

Rapidly changing national public health guidance Difficulty navigating easing of restrictions

Unexpected benefits

Working from home allowed for better management of pregnancy symptoms

No pressure from visitors in postnatal period More time with partner in "fourth trimester"

Pregnancy as a positive focus in an otherwise difficult time
Participant quotes

Q1: "It would have been nice to meet other mums who were due their baby at the same time. So all that social element was totally gone" [P10]

Q2 "every week my family are like "aw send us a picture of your bump" " P12

Q3: “ in the waiting room there was always a lovely atmosphere, it was gone after the Covid stuff hit" P10

Q1: "You can't interact and you can't ask anyone" P 11

Q2: "Suddenly the risks of the home birth became less than having to spend time in [hospital]" P8

Q3 "I don't think there was any change in the room, the midwives, anything like that. I didn't feel I had any less care" P2

Q4 "I just find that was much better in a home setting than in the hospital setting when there's so much going on" P1

Q5 "I didn't feel like his mother... my whole day was spent waiting for that 15 min" P2

Q1 "It was just really strange.. I had to go in on my own.. and..I was worried that something had happened to him [baby] and I had to go and sit in the waiting room by myself" $P 1$

Q2 'I didn't experience the 'not having your partner there'.. but my god it just terrifies me, the idea that I wouldn't have anybody that was on my side who knew me while being so very vulnerable which you are in labor" $P 8$

Q3 "it was a team effort and I felt that had been taken away by having to go to the appointments.. by myself" P10

Q4 "I would have benefitted hugely from having him being able to go to the NICU... He would have asked questions" P2

Q5 "It's this whole thing of... seeing partners as visitors as opposed to partners" P6

Q6 "chances are your birth partner is a member of your household" P3

Q1 “the reality is it's so new I don't think people know" [P11]

Q2 “Initially they were saying 'no pregnant women aren't any more high risk than anybody else' but then.. the HSE are saying to their own pregnant staff 'you shouldn't be working near coronavirus patients'” P11

Q3 "I'm getting different messages" P4

Q4 "the phrasing used was.. your birth partner will be allowed in when you're in active labor... like what does that mean?" P10

$\mathrm{Q} 1$ "We didn't have symptoms. We were just pregnant, but we didn't have asthma ... or cystic fibrosis. We were just pregnant women. And we were thinking, okay, so, [can] I still go on the Dart [commuter railway network] or will I still go on the bus? "P11

Q2 “It's harder to know the right thing to do is... what is being responsible, what is being paranoid" $P 9$

Q1 "We brought him home and my plan was to..lock the door, stay in... We definitely benefited from that." P1

Q2 “...not being overwhelmed with visitors. I'm quite glad that we can kind of have an excuse to say to people 'actually, not at the moment' " $P 9$

Q3 "I think.. one of the advantages to being pregnant.. is that you... feel like there's an end to it... psychological end... my family and friends are like it's groundhog day and I'm like no it's not because I've got a new milestone every week or a new thing to look forward to.. so that kind of helps" P9 pregnant women which added to a sense of isolation. Some felt there was less excitement surrounding the pregnancy. Changes to antenatal clinic visits made it an unpleasant experience for some women. Women had varying experiences of the changes in how care was delivered. One woman felt safe coming to a stand-alone maternity hospital as it felt 
removed from the pandemic which she associated with general hospitals. Some women had virtual or telephone consults which they felt de-personalized the experience with one woman describing a phone consultation with her obstetrician at 16 weeks as "tokenistic." One woman found a reduction in clinic visits increased her anxiety, as being in her first pregnancy; she felt she needed the reassurance of a hospital visit.

Many hospitals changed to provide antenatal classes online. One woman felt that doing pre-recorded classes took away from the experience as it was less individualized. One woman changed to a planned home birth at an advanced gestation due to fears about how the hospital would manage a potential outbreak. The three women who did experience hospital birth during the pandemic felt that the birth experience itself was not negatively impacted by the restrictions. Some hospitals expanded early transfer home services to reduce the capacity on the postnatal ward which women found beneficial.

One participant was directly affected by restrictions in NICU when her baby was unexpectedly admitted. For 2 weeks, she was limited to spending 15 min every day with her baby which was extremely difficult. Measures taken by the hospital to make this situation less stressful for her included daily text, picture, and video updates via smartphone.

One of the most distressing aspects of the change in care during the pandemic was the restrictions on partners attending hospital visits, particularly the anomaly scan. One woman described attending out of hours with reduced fetal movements and found this experience particularly difficult to go through alone. While none of the hospitals prevented partners from attending the birth of their children, the potential for tighter restrictions on partners caused a lot of anxiety. Women feared losing their birth partner as an advocate while they were in labor and after the birth. Women described that by partners missing out on appointments, he/she felt disconnected from the process.

Strict NICU restrictions during the initial lockdown meant that partners were not permitted to visit. One woman who was affected by these restrictions felt this added to the stress of having an unwell baby. Not having the baby's father present in the NICU added to concerns about how he would bond with the infant. The mother also felt more isolated without the support of her partner as an advocate or someone to ask questions on her behalf. One woman pointed out that partners were labelled as "visitors" when they should have been given special status. Many women could not understand the restrictions placed on partners attending when they were often from the same household and felt they should have been viewed as a family unit rather than as individuals.

Many women felt that by keeping informed, they would feel empowered but also appreciated that having too much access to news could feel overwhelming. The sources of information ranged from mainstream news and social media to peer-reviewed medical journals. The lack of upto-date pregnancy-specific information about the virus and its potential impact on pregnancy was a source of anxiety. Women also feared for the health of their babies and how they would manage to care for other children if they were to become unwell. They looked at hospital websites, but this was often not their primary source of information. The lack of relevant information meant that women were less likely to trust their healthcare providers as a source as they realized that the medical community was uncertain about the effects of COVID-19 in pregnancy. Conflicting information was a source of anxiety for women. Some of the messaging and language used around restrictions by the hospitals were unclear which caused stress amongst women, especially when information related to partners' presence on delivery suite.

Women received conflicting information about the potential impact of the virus on their health. Many women felt vulnerable and chose to cocoon despite the national recommendation that this was not necessary. While the initial "lockdown" message was clear, the gradual easing of restrictions brought new challenges.

The lockdown has some unexpected benefits. It gave many women the opportunity to work from home which made it easier to manage normal pregnancy symptoms such as fatigue or nausea and gave more time to devote to exercise, rest, or spending with their partners. This was also valuable after the birth as many women felt this extra time allowed for bonding and there was no pressure to see people or bring their new baby out. Many women's partners continued working from home after the birth which allowed for additional support in the "fourth trimester." Many women appreciated quieter waiting rooms and benefitted from more community-based care including early transfer home teams. One woman described the positive milestones in her pregnancy as a welcome distraction from the pandemic.

\section{Discussion}

The disruption and uncertainty that came with the pandemic caused great anxiety at a time when pregnant women were feeling particularly vulnerable. While understandably a lot of initial research into COVID-19 in pregnancy was on the physical and medical aspects, we are beginning to see the psychological effects that isolation and prolonged restrictions are having on pre- and postnatal psychological wellbeing and depression [18].

We know women have been disproportionately affected by the social and economic consequences of the pandemic [19]. Several studies have now looked at the experiences of pregnant and postnatal women globally, and while their 
experiences are specific to their location and the timeframe in the pandemic that the research was carried out, recurrent themes are emerging. As with the women in our study, anxiety and fear [14, 20-24] were commonly reported as was the need for reliable information [22-24]. In one study of perinatal anxiety in the USA, $60 \%$ of women reported moderate or severe anxiety symptoms [25]. In a study in Italy, which was the epicenter of the pandemic in Europe in the early stages, women with a history of anxiety or depression were at a higher risk of anxiety symptoms or post-traumatic stress disorder during "lockdown" than those without[13]. Disruption to antenatal and postnatal care was seen all over the world [24, 26-28]; however the degree of disruption varied according to the resources, or lack of resources, in that particular country. Low- and middle-income countries are particularly vulnerable to the disruption of routine maternal and neonatal care [27, 29].

Most women in our study sought information as a coping strategy, often from social media or their peers rather than from the hospital itself. When the government in Ireland advised groups considered at risk to "cocoon," pregnant women were not included on this list, in contrast to guidance in the UK. This caused confusion in our cohort as women were often sourcing their information from the UK. In an international cross-sectional study, a lack of information about COVID-19 in pregnancy was noted to exacerbate fears among women of the risks of the disease in pregnancy [30]. In a study in China, 90\% of women questioned considered themselves vulnerable from the effects of COVID-19 [31]. As a result of this perceived risk and the potential for the spread of infection from birth and through breastfeeding, they reported increased rates of requests for planned caesarean birth and higher rates of bottle feeding [31]. While there was no association between information source and level of anxiety in that study, we know that conflicting information in times of humanitarian crises is associated with higher levels of stress [32]. The adverse effects of maternal stress on the fetus and on infant cognitive development are well documented [33-35].

Due to the time-sensitive nature of pregnancy, maternity services had to quickly adapt and pivot to continue to provide care to an acceptable standard. Some aspects of maternity care such as assessing fetal wellbeing cannot adequately be carried out remotely. Some of the strategies adopted by maternity hospitals were to introduce less frequent antenatal appointments and carry out some consultations by telephone [2]. While the women interviewed were largely accepting of the need for restrictions, some felt these changes lead to deficiencies in their care. In one study on women's experiences of pregnancy during COVID-19 restrictions in Australia, concerns were voiced over telehealth which women felt was impersonal and care felt rushed [21]. While there is a role for developing telemedicine, it is important not to depersonalize care. There is a need for enhanced online support [31] and greater investment to make use of available technologies to improve the antenatal experience for women.

The women in this study relied on their partners for emotional support. The perceived risk that a birth partner would not be present for delivery was a huge source of anxiety. This has been a key finding in several studies in this area [21, 23, 36]. The presence of a supportive birth partner is associated with favorable birth outcomes [37]. Indeed, the World Health Organization (WHO) has upheld that all pregnant women should have a companion of their choice present during birth [38]. In one study of the effects of COVID-19 on women's birth expectations, women with a history of mental health disorders expressed greater concern for their partner's health highlighting the important role a partner can have during the pregnancy, birth, and postnatal period [39]. The women interviewed also felt a sense of isolation from friends, family members, and the lack of opportunity to meet other pregnant women which has similarly been noted in other studies [40]. This lack of social support was a significant contributor to perinatal stress during the pandemic (30).

Despite the difficult circumstances, women were, perhaps surprisingly, able to find some positive aspects in their situation. Reduced appointments meant quieter waiting rooms and additional community supports allowed for healthy women to be discharged home earlier postnatally. The ability to work from home while pregnant was a big advantage, eliminating commuting and allowing women to rest and work in their own time while maintaining productivity. Women also benefitted from having their partners work from home in the postpartum period as they were able to provide additional support. This is something that employers may consider going forward as we navigate a "new normal."

Most of the available literature in this area has been gathered through a survey which allows for larger cohorts. The strength of our study is in the in-depth interviews which took place at the height of restrictions, offering a unique perspective on pregnancy in the pandemic and adding to the growing narrative of the voices of women who gave birth during a global health crisis. This study is limited by the lack of ethnic diversity in the group interviewed despite attempts to reach out to minority groups. Further research is needed on the impact of the pandemic on minority groups.

\section{Conclusion}

The COVID-19 pandemic is a time of great anxiety for pregnant women. This was exacerbated in the beginning by uncertainty about the effects of COVID-19 on pregnancy and unclear messaging about restrictions. The birth partner is an important role as an advocate for women and should be included in antenatal care. Containment strategies for the pandemic should be developed with this in mind and attempt 
to view the family as a unit rather than the woman in isolation. Going forward there is an opportunity to expand on community delivered care, particularly for low-risk women and an urgent need to improve mental health support to deal with the potential long-term sequelae of this pandemic.

Author contribution NK has made a substantial contribution to the conception and design of the work, the acquisition analysis, and interpretation of the data; has drafted the work; and has approved the submitted version and agrees to be both personally accountable for her contribution and to ensure that questions related to the accuracy and integrity of any part of the work even ones in which the author was not personally involved are appropriately investigated and resolved and the resolution is documented in the literature. BD has made a substantial contribution to the conception of the work, to the interpretation of the data, has substantially revised it, and has approved the submitted version and agrees to be both personally accountable for his contribution and to ensure that questions related to the accuracy and integrity of any part of the work even ones in which the author was not personally involved are appropriately investigated and resolved and the resolution is documented in the literature. SC has made a substantial contribution to the conception of the work and to the interpretation of the data, has substantially revised it; and has approved the submitted version and agrees to be both personally accountable for her contribution and to ensure that questions related to the accuracy and integrity of any part of the work even ones in which the author was not personally involved are appropriately investigated and resolved and the resolution is documented in the literature. FMcA has made a contribution to the conception and design of the work and to the interpretation of the data, has revised it; and has approved the submitted version and agrees to be both personally accountable for her contribution and to ensure that questions related to the accuracy and integrity of any part of the work even ones in which the author was not personally involved are appropriately investigated and resolved and the resolution is documented in the literature. JL has made a substantial contribution to the conception and design of the work and to the interpretation of the data; has substantially revised it; and has approved the submitted version and agrees to be both personally accountable for her contribution and to ensure that questions related to the accuracy and integrity of any part of the work even ones in which the author was not personally involved are appropriately investigated and resolved and the resolution is documented in the literature. MFH has made a substantial contribution to the conception and design of the work, the acquisition analysis, and interpretation of the data; has drafted the work; and has approved the submitted version and agrees to be both personally accountable for her contribution and to ensure that questions related to the accuracy and integrity of any part of the work even ones in which the author was not personally involved are appropriately investigated and resolved and the resolution is documented in the literature.

Data availability The anonymized qualitative data set, analyzed during the current study, is available from the corresponding author upon reasonable request and Research Ethics approval.

Code availability Not applicable.

\section{Declarations}

Ethics Ethical approval was granted by the National Maternity Hospital Research Ethics Committee (EC 14.2020). Women were provided with written information and informed consent was obtained from all participants prior to participation.
Consent to participate All participants gave written consent to participate in this study.

Consent for publication All participants gave written consent for the anonymous publication of quotes from interviews.

Conflict of interest The authors declare no competing interests.

\section{References}

1. WHO (2020) Statement on the second meeting of the International Health Regulations (2005) Emergency Committee regarding the outbreak of novel coronavirus (2019-nCoV) 2020 [Available from: https://www.who.int/news/item/30-01-2020-statementon-the-second-meeting-of-the-international-health-regulations(2005)-emergency-committee-regarding-the-outbreak-of-novelcoronavirus-(2019-ncov)

2. Sheil O, McAuliffe FM (2021) Reorganisation of obstetric services during the COVID pandemic - experience from National Maternity Hospital Dublin Ireland. Best Practice \& Res Clinical Obstetrics \& Gynaecol

3. Liu Y, Chen H, Tang K, Guo Y (2020) Clinical manifestations and outcome of SARS-CoV-2 infection during pregnancy. J Infect

4. Schwartz DA, Graham AL (2020) Potential maternal and infant outcomes from (Wuhan) coronavirus 2019-nCoV infecting pregnant women: lessons from SARS, MERS, and other human coronavirus infections. Viruses 12(2)

5. Allotey J (2021) Update to living systematic review on covid-19 in pregnancy. BMJ 372:n615

6. Zarocostas J (2020) How to fight an infodemic. Lancet 395(10225):676

7. Han PKJ, Zikmund-Fisher BJ, Duarte CW et al (2018) Communication of scientific uncertainty about a novel pandemic health threat: ambiguity aversion and its mechanisms. J Health Commun 23(5):435-444

8. Lin L, Savoia E, Agboola F, Viswanath K (2014) What have we learned about communication inequalities during the H1N1 pandemic: a systematic review of the literature. BMC Public Health 14:484

9. Perrin PC, McCabe OL, Everly GS Jr, Links JM (2009) Preparing for an influenza pandemic: mental health considerations. Prehosp Disaster Med 24(3):223-230

10. Harville E (2010) Disasters and perinatal health : a systematic review. Obstet Gynecol Surv 65:713-728

11. Goodman LA, Salyers MP, Mueser KT et al (2001) Recent victimization in women and men with severe mental illness: prevalence and correlates. J Trauma Stress 14(4):615-632

12. Marjanovic Z, Greenglass ER, Coffey S (2007) The relevance of psychosocial variables and working conditions in predicting nurses' coping strategies during the SARS crisis: an online questionnaire survey. Int J Nurs Stud 44(6):991-998

13. Ravaldi C, Ricca V, Wilson A et al (2020) Previous psychopathology predicted severe COVID-19 concern, anxiety, and PTSD symptoms in pregnant women during "lockdown" in Italy. Arch Womens Ment Health

14. Ceulemans M, Foulon V, Ngo E et al (2021) Mental health status of pregnant and breastfeeding women during the COVID-19 pandemic-a multinational cross-sectional study. Acta Obstet Gynecol Scand

15. Biaggi A, Conroy S, Pawlby S, Pariante CM (2016) Identifying the women at risk of antenatal anxiety and depression: a systematic review. J Affect Disord 191:62-77 
16. O'Brien BC, Harris IB, Beckman TJ et al (2014) Standards for reporting qualitative research: a synthesis of recommendations. Acad Med 89(9):1245-1251

17. Glaser B (1978) Theoretical Sensitivity. Advances in the Methodology of Grounded Theory

18. Berthelot N, Lemieux R, Garon-Bissonnette J et al (2020) Uptrend in distress and psychiatric symptomatology in pregnant women during the coronavirus disease 2019 pandemic. Acta Obstet Gynecol Scand 99(7):848-855

19. Burki $\mathrm{T}$ (2020) The indirect impact of COVID-19 on women. Lancet Infect Dis 20(8):904-905

20. Sahin BM, Kabakci EN (2021) The experiences of pregnant women during the COVID-19 pandemic in Turkey: a qualitative study. Women and Birth 34(2):162-169

21. Atmuri K, Sarkar M, Obudu E, Kumar A (2021) Perspectives of pregnant women during the COVID-19 pandemic: A qualitative study. Women Birth

22. Barbosa-Leiker C, Smith CL, Crespi EJ et al (2021) Stressors, coping, and resources needed during the COVID-19 pandemic in a sample of perinatal women. BMC Pregnancy Childbirth 21(1):171

23. Sweet L, Bradfield Z, Vasilevski V et al (2021) Becoming a mother in the 'new' social world in Australia during the first wave of the COVID-19 pandemic. Midwifery 98:102996

24. Mortazavi F, Ghardashi F (2021) The lived experiences of pregnant women during COVID-19 pandemic: a descriptive phenomenological study. BMC Pregnancy Childbirth 21(1):193

25. Farewell CV, Jewell J, Walls J, Leiferman JA (2020) A mixedmethods pilot study of perinatal risk and resilience during COVID19. J Prim Care Community Health 11:2150132720944074

26. Karavadra B, Stockl A, Prosser-Snelling E et al (2020) Women's perceptions of COVID-19 and their healthcare experiences: a qualitative thematic analysis of a national survey of pregnant women in the United Kingdom. BMC Pregnancy Childbirth 20(1):1-8

27. Hailemariam S, Agegnehu W, Derese M (2021) Exploring COVID-19 related factors influencing antenatal care services uptake: a qualitative study among women in a rural community in Southwest Ethiopia. J Prim Care Community Health 12:2150132721996892

28. Javaid S, Barringer S, Compton SD et al (2021) The impact of COVID-19 on prenatal care in the United States: qualitative analysis from a survey of 2519 pregnant women. Midwifery 98:102991

29. Reinders S, Alva A, Huicho L, Blas MM (2020) Indigenous communities' responses to the COVID-19 pandemic and consequences for maternal and neonatal health in remote Peruvian Amazon: a qualitative study based on routine programme supervision. BMJ open 10(12):e044197

30. Meaney S, Leitao S, Olander EK et al (2021) The impact of COVID-19 on pregnant womens' experiences and perceptions of antenatal maternity care, social support, and stress-reduction strategies. Women and Birth

31. Liu X, Chen M, Wang Y et al (2020) Prenatal anxiety and obstetric decisions among pregnant women in Wuhan and Chongqing during the COVID-19 outbreak: a cross-sectional study. BJOG 127(10):1229-1240

32. Purgato M, Gastaldon C, Papola D et al (2018) Psychological therapies for the treatment of mental disorders in low- and middle-income countries affected by humanitarian crises. Cochrane Database Syst Rev 7:CD011849

33. Wu Y, Lu YC, Jacobs M et al (2020) Association of prenatal maternal psychological distress with fetal brain growth, metabolism, and cortical maturation. JAMA Netw Open 3(1):e1919940

34. Madigan S, Oatley H, Racine N, Fearon RMP, Schumacher L, Akbari E et al (2018) A meta-analysis of maternal prenatal depression and anxiety on child socioemotional development. J Am Acad Child Adolesc Psy 57(9):645-57 e8

35. Tarabulsy GM, Pearson J, Vaillancourt-Morel MP et al (2014) Meta-analytic findings of the relation between maternal prenatal stress and anxiety and child cognitive outcome. J Dev Behav Pediatr 35(1):38-43

36. Cullen S, Doherty J, Brosnan M (2021) Women's views on the visiting restrictions during COVID-19 in an Irish maternity hospital. Br J Midwifery 29(4):216-223

37. Bohren MA, Hofmeyr GJ, Sakala C, Fukuzawa RK, Cuthbert A (2017) Continuous support for women during childbirth. Cochrane Database Sys Rev (7)

38. Organisation WH (2020) Coronavirus disease (COVID-19): Pregnancy and childbirth 2020 [Available from: https://www.who.int/ news-room/q-a-detail/coronavirus-disease-covid-19-pregnancyand-childbirth

39. Ravaldi C, Wilson A, Ricca V et al (2020) Pregnant women voice their concerns and birth expectations during the COVID-19 pandemic in Italy. Women Birth

40. Chivers BR, Garad RM, Boyle JA et al (2020) Perinatal distress during COVID-19: thematic analysis of an online parenting forum. J Med Internet Res 22(9):e22002

Publisher's Note Springer Nature remains neutral with regard to jurisdictional claims in published maps and institutional affiliations. 